\title{
Paradigm Shift: From Standard-Driven Protocols to Resource-Driven Guidelines for Neurotrauma Man- agement in Low- and Middle-Income Countries
}

\author{
Tariq Khan ${ }^{1}$ Mukhtar Khan ${ }^{1}$ \\ ${ }^{1}$ Department of Neurosurgery, Northwest School of Medicine and
Northwest General Hospital, Peshawar, Pakistan
}

J Neurosci Rural Pract 2020;11:5-6

The project by Rubiano et al known as BOOTStraP ${ }^{1}$ is part of an effort toward provision of resource-stratified guidelines for the management of neurotrauma in low-resource settings. This article, which is aptly acronymed as "BOOTStraP," is a landmark work by Rubiano et al, has combined evidence-based guidelines with consensus-based recommendations from experts in trauma management in general and neurotrauma management in particular. The current work stems out from the collaborative efforts of the Colombian Association of Neurosurgery, the World Federation of Neurosurgical Societies (WFNS), and the Global Health Research Group for Neurotrauma (GHRGN) of the National Institute of Health Research (NIHR). The BOOTStraP is a timely effort in the context of rising awareness of the dire situation of poor patient outcomes in low- and middle-income countries (LMICs), and we are sure that it will be of help in improving neurotrauma care in the LMICs. One of the unique features of the BOOTStraP is that it focuses on all aspects of the needs of different situations in management of neurotrauma, ranging from prevention to advanced neurointensive care for mild-to-severe TBI and TBI in polytrauma settings.

Significant advancements have been made in prevention and care of TBI patients over the last three decades. These advancements are better understanding of TBI pathophysiology, improved therapeutics, accurate monitoring modalities, timely and evidence-based surgical interventions, and novel neurorehabilitation strategies. Collectively, all these advancements have led to improved patient care in the high-income countries (HICs). However, the LMICs clearly lag behind the HICs in both availability of medical resources (workforce, equipment, facilities, transport systems, acute and intensive care, and rehabilitation), as well as implementation of preventive, and surgical care policies. ${ }^{2}$ Delays and lack of timely access to good quality care for neurotrauma patients worsen morbidity and mortality, and this fact becomes important for time-critical conditions such as neurotrauma. ${ }^{3}$ One important factor as identified by Kruk et $\mathrm{al}^{4}$ in a recently published study was the lack of quality health care. They have even stressed that mere availability of health care access (of a poor quality) may lead to increased morbidity and mortality and have advocated the use of quality health care access as opposed to the mere availability of health care access. The BOOTStraP has tried in its entirety to address the issue of improving quality health care provision for TBI patients and not just provision of care. To our understanding, and as evident from several recent studies, the poor outcome of TBI patients in LMICs might be due to a totally different set of requirements which in HICs might not even be perceived to be. ${ }^{5,6}$ One example is the nonavailability of intravenous fluids at far-flung areas in rural LMICs or the lack of a proper rescue and transport system which might not be the case in HICs. ${ }^{7}$ The BOOTStraP identifies these barriers and addresses situation-wise solutions, as to what should be done. This indicates that there is a need for a paradigm shift from standard-driven protocol to a standard of care, which is catering for the needs of limited resource settings as well.

It is clear that LMICs lag far-behind the HICs in various aspects of surgical capacity and especially, the neurosurgical workforce. ${ }^{8}$ LMICs have one of the highest incidences of TBI and traumatic spinal cord injury (90\% of the global incidence). More than half of the total caseload that a neurosurgeon faces is neurotrauma (TBI and spinal cord injury) that is estimated to be 5 million neurosurgical procedures per year in the LMICs. ${ }^{9}$ However, it is evident that a large number of LMICs either do not have a single neurosurgeon, or are very low in number working at main urban centers. In comparison to this, the HICs have an impressive neurosurgeon-to-patient ratio. The gamut of this disparity of lower resources and higher incidence of neurotrauma falls on the communities and has tremendous global impact in terms of disability
Address for correspondence

Tariq Khan, FRCS, Department of Neurosurgery,

Northwest School of Medicine and Northwest

General Hospital, Peshawar, Pakistan

(e-mail: tariqhashimkhan@hotmail.com).
DOI https://doi.org/

10.1055/s-0040-1701558 ISSN 0976-3147.
(C)2020 Association for Helping Neurosurgical Sick People
License terms

$($ () (1) $\Theta \circledast$ 
and death. The WHO's "Sustainable Development Goal 3.6" states that in order to make a significant global impact, a 50\% reduction in injuries and deaths from road traffic injuries (RTIs) will be required by $2030 .{ }^{10}$ The BOOTStraP paper is the best example of one step forward in this direction.

During the 68th World Health Assembly in 2015, the member states of the World Health Organization unanimously passed a resolution, which called for "strengthening emergency and essential surgical care and anesthesia as a component to universal health coverage." The report of the Lancet Commission of Global Surgery showed that surgical disease burden was in fact greater than the problem of communicable diseases and can only be solved by strengthening surgical health care in the LMICs. ${ }^{11}$

Recently, with increasing interest in addressing barriers to health care in LMICs, a major area for study was the lack of resource-stratified guidelines that can be applicable to needs of trauma patients in general and neurotrauma in particular. ${ }^{12}$ These resource stratified guidelines are essential for improving the overall strength of the surgical health care system ranging from rescue and prehospital care to acute care and rehabilitation, so that no individual suffers undue disability due to lack of emergency neurotrauma care.

As part of the strides toward strengthening surgical systems, the global movement of provision of national surgical, obstetrics, and anesthesia plans (NSOAPs) was launched in the developing countries. This was followed by launching of provincial or state-level surgical, obstetrics and anesthesia plans (PSOAPs). On a continuum to the commitment of the WFNS and the NIHR GHRGN, and in collaboration with the scholarly cooperation of the Harvard medical school, the United States, and Northwest School of Medicine, Peshawar, Pakistan, the Neurotrauma Committee of WFNS launched the "Comprehensive Policy Recommendations for the Management of Brain \& Spine Injuries in Lower \& Middle Income Countries(Peshawar recommendations)" and provide the policy makers and stakeholders with the minimal standards needed for care of neurotrauma patients, as well as identifying deficits, in care provision. ${ }^{13}$ These guidelines will also help to present the policy makers a direction toward goals for enhancing the quality of neurotrauma care.

The next step to move toward improvement of neurotrauma care would be the testing phase of the BOOTStraP recommendations, and we are sure that it will help in improving neurotrauma outcomes in LMICs. Once the recommendations are validated externally, we would suggest to translate these in multiple languages, publish it as booklet, and disseminate it to the care providers at various levels involved in care of TBI patients. An additional effort that we must advocate for is the inclusion of the BOOTStraP recommendations within the Peshawar recommendations framework, so that it can further be the work on quality neurotrauma care.

\section{Conflict of Interest}

None declared.

\section{References}

1 Rubiano AM, Vera DS, Montenegro JH, et al. Recommendations of the Colombian consensus committee for the management of traumatic brain injury in prehospital, emergency department, surgery, and intensive care (beyond one option for treatment of traumatic brain injury, a stratified protocol (BOOTStraP). J Neurosci Rural Pract 2020;11(1):7-22

2 Rubiano AM, Carney N, Chesnut R, Puyana JC. Global neurotrauma research challenges and opportunities. Nature 2015;527(7578):S193-S197

3 Bashford T, Joannides A, Phuyal K, et al. Nuancing the need for speed: temporal health system strengthening in low-income countries. BMJ Glob Health 2019;4(4):e001816

4 Kruk ME, Gage AD, Joseph NT, Danaei G, García-Saisó S, Salomon JA. Mortality due to low-quality health systems in the universal health coverage era: a systematic analysis of amenable deaths in 137 countries. Lancet 2018;392(10160):2203-2212

5 Punchak M, Mukhopadhyay S, Sachdev S, et al. Neurosurgical care: availability and access in low-income and middle-income countries. World Neurosurg 2018;112:e240-e254

6 Rubiano AM, Puyana JC, Mock CN, Bullock MR, Adelson PD. Strengthening neurotrauma care systems in low and middle income countries. Brain Inj 2013;27(3):262-272

7 Chesnut RM. Intracranial pressure monitoring: headstone or a new head start. The BEST TRIP trial in perspective. Intensive Care Med 2013;39(4):771-774

8 Corley J, Lepard J, Barthélemy E, Ashby JL, Park KB. Essential neurosurgical workforce needed to address neurotrauma in low- and middle-income countries. World Neurosurg 2019;123:295-299

9 Kumar R, Lim J, Mekary RA, et al. Traumatic spinal injury: global epidemiology and worldwide volume. World Neurosurg 2018;113:e345-e363

10 World Health Organization. Sustainable development gaols (SDGs): Goal 3. Target 3.6 : By 2020, halve the number of global deaths and injuries from road traffic accidents. [poster] 2016. WHO. Manila: WHO Regional Office for the Western Pacific. Available at: http://iris.wpro.who.int/handle/10665.1/12878. Accessed January 23, 2020

11 Meara JG, Leather AJ, Hagander L, et al. Global surgery 2030: evidence and solutions for achieving health, welfare, and economic development. Surgery 2015;158(1):3-6

12 Dewan MC, Rattani A, Fieggen G, et al. Global neurosurgery: the current capacity and deficit in the provision of essential neurosurgical care. Executive Summary of the Global Neurosurgery Initiative at the Program in Global Surgery and Social Change. J Neurosurg 2018 (e-pub ahead of print). doi: 10.3171/2017.11.JNS171500

13 Corley J, Barthèlemy EJ, Lepard J, et al. Comprehensive policy recommendations for head and spine injury care in low- and middle-income countries. World Neurosurg 2019;132:434-436 